\title{
Medullary Thyroid Cancer Patient's Assessment of Quality of Life Tools: Results from the QaLM Study
}

\author{
Laura Moss $^{\mathrm{a}}$ Catrin Cox ${ }^{\mathrm{b}}$ Jonathan Wadsley ${ }^{\mathrm{c}}$ Kate Newbold ${ }^{\mathrm{d}}$ \\ Mark W.J.Strachan ${ }^{\text {e }}$ Maralyn Druce ${ }^{f}$ Neil Tolley $^{g}$ Kathryn Graham ${ }^{\text {h }}$ \\ Sarah Jefferies ${ }^{\mathrm{i}}$ Lydia Fresco $^{\mathrm{j}}$ Suganya Sivabalasingham ${ }^{\mathrm{k}}$ \\ Alistair Balfour' Chris Hurt $^{\mathrm{b}}$
}

${ }^{a}$ Velindre Cancer Centre, Velindre University NHS Trust, Cardiff, UK; ${ }^{b}$ Centre for Trials Research, Cardiff University, Cardiff, UK; ' ${ }^{C}$ Weston Park Cancer Centre, Sheffield, UK; ${ }^{d}$ The Royal Marsden NHS Foundation Trust, London, UK; eWestern General Hospital, Edinburgh, UK; ${ }^{f}$ Barts and the London School of Medicine and Dentistry, London, UK; 9 Imperial College Healthcare NHS Trust, London, UK; ${ }^{h}$ Beatson Oncology Centre, Glasgow, UK; 'Cambridge University Hospitals NHS Foundation Trust, Cambridge, UK; jUniversity Hospitals Coventry and Warwickshire NHS

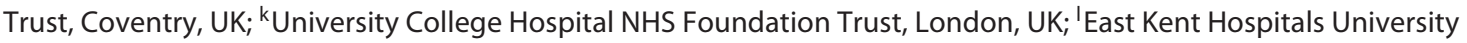
NHS Foundation Trust, Canterbury, UK

\section{Keywords}

Medullary thyroid cancer · Neuroendocrine tumour ·

Quality of life · Questionnaires

\begin{abstract}
Background: Medullary thyroid cancer (MTC) is a neuroendocrine tumour and a rare variant of thyroid cancer with different aetiology, presentation and treatment to differentiated thyroid cancer. Currently available thyroid cancer-specific quality of life (QoL) tools focus on issues and treatments more relevant to patients with differentiated thyroid cancer and therefore may not address issues specific to a MTC diagnosis and cancer journey. Method: This prospective multicentre randomised study involved 204 MTC patients completing four quality of life questionnaires (QOLQ) and stating their most and least preferred. The questionnaires were a
\end{abstract}

general instrument, the EORTC QLQ-C30, two disease-specific tools, the MD Anderson Symptom Inventory (MDASI) thyroid module and the City of Hope Quality of Life Scale/ THYROID (amended) and the neuroendocrine questionnaire, EORTC QLQ-GINET21. Patients were randomised to complete the four questionnaires in one of 24 possible orders and then answered questions about which tool they preferred. The primary outcome measure was patients' preferred QoL instrument for describing their concerns and for facilitating communication with their healthcare professional. Secondary analyses looked at differences between preferred QOLQs amongst patient subgroups (WHO performance status [0 and 1+], disease stage: early [T1-3, N0 or $\mathrm{N} 1 \mathrm{~A}]$, metastatic [T4, any $\mathrm{T} \mathrm{N} 1 \mathrm{~b}$ ] and advanced [any $\mathrm{T}$ any $\mathrm{N}$ M1], and type of MTC [sporadic and inherited]), identification of MTC patients' least preferred questionnaire and clinicians' views on the QoL tools in terms of their ability to highlight
Laura Moss

Clinical Oncology Department Velindre Cancer Centre, Velindre Road Glamorgan, Cardiff CF14 2TL (UK)

laura.moss@wales.nhs.uk 
problems not otherwise ascertained by a standard clinical review. Results: No evidence of a difference was observed for most preferred QOLQ $(p=0.650)$. There was however evidence of a difference in least preferred questionnaire in the cohort of 128 patients who stated their least preferred questionnaire $(p=0.042)$, with $36 \%(46 / 128)$ of patients choosing the EORTC QLQ-GI.NET21 questionnaire. Subgroup analyses showed that there was no evidence of a difference in patients' most preferred questionnaire in sporadic MTC patients $(p=0.637)$, patients with WHO PS 0 or $1+(p=0.844$ and $p=0.423$ ) nor when comparing patients with early, advanced local or metastatic disease $(p=0.132, p=0.463$ and $p=0.506$, respectively). Similarly, subgroup analyses on patients' least preferred questionnaires showed no evidence of differences in sporadic MTC patients $(p=0.092)$, patients with WHO PS 0 or $1+(p=0.423$ and $p=0.276)$, nor in early or metastatic disease patients ( $p=0.682$ and $p=0.345$, respectively). There was however some evidence to suggest a difference in least preferred questionnaire in patients with advanced local stage disease $(p=0.059)$, with $43 \%(16 / 37)$ of these patients choosing the EORTC QLQ-GI.NET21 questionnaire. Conclusions: MTC patients regardless of their performance status, disease aetiology and disease burden did not express a preference for any one particular questionnaire suggesting any of the tools studied could be utilized in this patient cohort. The least preferred questionnaire being a gastrointestinal NET specific tool suggests that diarrhoea was not a significant symptom and concern for the population studied.

(C) 2020 European Thyroid Association Published by S. Karger AG, Basel

\section{Introduction}

Medullary thyroid cancer (MTC) is a rare variant of thyroid cancer with different aetiology, presentation, treatment and symptoms compared to the more common differentiated thyroid cancers (DTC). Based on Cancer Research UK (CRUK) 2014-2016 statistical data [1] and an estimated 4\% [2] of all thyroid cancers being MTC, we would expect approximately 140 new cases of MTC per year in the UK with a 10-year disease-specific survival rate of approximately $75 \%$ [2].

MTC presents as a sporadic tumour in approximately $75 \%$ of cases and as an inherited disease in 25\% [3]. Inherited cases are associated with multiple endocrine neoplasia type 2A (MEN2A), multiple endocrine neoplasia type 2B (MEN2B), and familial MTC (FMTC). Patients with MEN2A and MEN2B have additional, associated medical conditions, e.g., phaeochromocytoma and hy-

Assessment of Quality of Life Tools in

Medullary Thyroid Cancer Patients perparathyroidism, which can impact on their quality of life (QoL).

Patients with rare cancers can face additional barriers and concerns including limited patient information resources, the potential for lack of understanding amongst family and friends and healthcare professionals who do not have experience of managing their rare condition. It can be difficult to fully appreciate the impact of a cancer diagnosis (particularly of a rare type or as part of an inherited syndrome), raised and rising tumour markers and disease symptoms on a patient's QoL.

QoL is multi-faceted and is affected by emotional, social and functional issues as well as disease-related problems. QoL tools can increase healthcare professionals' understanding of the most important issues for a patient thus facilitating the consultation as well as improving the patient's satisfaction with the consultation.

Currently available thyroid cancer-specific QoL tools tend to be focused on issues and treatments more relevant to patients with DTC rather than MTC. MTC is a neuroendocrine tumour (NET) and patients with bulky local or distant disease often have other symptoms, such as diarrhoea, which are not addressed by the currently available thyroid cancer QoL questionnaires.

The aim of the QaLM study (Assessment of Quality of Life Tools in Medullary Thyroid Cancer) was to determine which Health Related Quality of Life (HRQoL) instruments patients with sporadic and inherited MTC and early and advanced stage MTC find most helpful in facilitating communication of their health problems to their clinicians. Patients were asked to rank the questionnaires, and it was the rankings that were analysed. The study attempted to ascertain if this patient cohort's symptoms and QoL was better assessed by a thyroid cancer specific questionnaire, a general symptom questionnaire or a neuroendocrine tumour specific questionnaire. The study did not assess and compare the individual patient scores on each of the questionnaires.

The study also obtained clinician's views on the QoL tools in terms of their ability to highlight problems not otherwise ascertained by a standard clinical review appointment.

\section{Materials and Methods}

The QaLM study (NCT 02465424) was a multi-centre crosssectional study with randomisation undertaken in 17 centres in the UK.

The study utilised general QoL instruments as well as disease specific ones. The general instrument was the EORTC QLQ-C30 
questionnaire [4], and the disease-specific tools were the MD Anderson Symptom Inventory (MDASI) thyroid module [5] and the City of Hope Quality of Life Scale/THYROID (amended) [6] which both assess thyroid cancer issues and EORTC QLQ-GINET21 [7] which assesses issues relevant to neuroendocrine tumours. The City of Hope Scale was amended by removing four questions related to radioactive iodine treatment which is not relevant to the study population.

Inclusion criteria were patients 16 years old or over with a diagnosis of MTC (inherited or sporadic). Patients could be included at any time point after diagnosis. Patients with differentiated (papillary or follicular) thyroid cancer, anaplastic thyroid cancer or lymphoma of the thyroid were excluded.

Patients were randomised to complete the four QoL questionnaires in one of the 24 possible orders and then answered questions about which questionnaire they preferred. The questionnaires were presented in random order to counterbalance the effects of boredom and habituation. A researcher was available to assist with the completion of the questionnaires when required. Patients were able to insert additional comments as free text at the end of the final questionnaire.

Principal investigators were contacted at the end of the study to ascertain their views on the four QoL tools. They were asked whether any of the QoL tools had helped the patient prioritise concerns at subsequent clinic appointments. Investigators were not asked to rank the questionnaires as the focus of the study was on patient preferences.

The primary outcome was the identification of MTC patients' preferred QoL instrument for describing their concerns and for facilitating communication with their healthcare professional. This was measured by noting their most preferred questionnaire in terms of helpfulness. Secondary analyses looked at differences between preferred QoL tools amongst patient subgroups (WHO performance status [0 and $1+]$, disease stage: early [T1-3, N0 or $\mathrm{N} 1 \mathrm{~A}]$, metastatic [T4, any T N1b] and advanced [any T any N M1], and type of MTC [sporadic and inherited]), identification of MTC patients' least preferred questionnaire and clinicians' views on the QoL tools in terms of their ability to highlight problems not otherwise ascertained by a standard clinical review.

\section{Statistical Analysis}

All statistical analyses were conducted using Stata 14 according to a pre-specified analysis plan. A Pearson goodness-of-fit test was used on the most preferred questionnaire for each patient, with the assumption that each questionnaire was equally likely to be most preferred, and thus that the four QoL tools were equally useful in facilitating the communication of health concerns of MTC. A critical value of 0.4 , i.e., if one of the questionnaires was most preferred by $40 \%$ of the patients and setting $\alpha=0.05$, gave a sample size of 66 patients. It was estimated that approximately $35 \%$ of patients would have incomplete data and therefore a minimum of 100 patients would need to be recruited. A Pearson goodness-of-fit test was also used on the least preferred questionnaire for each patient to determine whether any of the questionnaires were significantly least preferred. This method was repeated to carry out subgroup analyses which assessed whether there were differences between the most and least preferred QoL tools amongst inherited MTC and sporadic MTC patients, early, advanced local, and metastatic stage disease patients and patients with WHO PS 0 and $1+$.

\section{Results}

A total of 204 patients were recruited from 17 sites between December 2015 and January 2018. Of the 144 out of 204 recruited patients contributing preference data to the study, 78\% (112/144) provided their most preferred questionnaire and $89 \%(128 / 144)$ provided their least preferred questionnaire. Age, gender, MTC type and TNM stage distributions were well balanced between participants that provided their most preferred QoL tool on the debriefing questionnaire $(n=112)$ and those that did not $(n=92)$ (Appendix: Table 1$)$.

For the patient cohort who provided their most preferred questionnaire $(n=112)$, there was no evidence of a difference in most preferred QoL questionnaire $\left(\chi^{2}=\right.$ $1.64, p=0.650$ ) (Appendix: Table 2). Subgroup analyses showed that there was no evidence of a difference in patients' most preferred questionnaire in sporadic MTC patients $\left(\chi^{2}=1.70, p=0.637\right)$, patients with WHO PS 0 or $1+\left(\chi^{2}=0.82, p=0.844\right.$ and $\left.\chi^{2}=2.80, p=0.423\right)$, nor when comparing patients with early, advanced local or metastatic disease $\left(\chi^{2}=5.62, p=0.132, \chi^{2}=2.57, p=\right.$ 0.463 and $\chi^{2}=2.33, p=0.506$, respectively) (Appendix: Table 3). There was however evidence of a difference in least preferred questionnaire in the cohort of $128 \mathrm{pa}$ tients who stated their least preferred questionnaire $\left(\chi^{2}=8.19, p=0.042\right)$, with $36 \%(46 / 128)$ of patients choosing the EORTC QLQ-GI.NET21 questionnaire compared to $21 \%(27 / 128), 22 \%(28 / 128)$ and $21 \%$ (27/128) of patients who least preferred EORTC QLQC30, MDASI-Thy and City of Hope: QoL-Thy, respectively (Appendix: Table 2). When carrying out subgroup analyses on patients' least preferred questionnaires, no evidence of differences were found in sporadic MTC patients $\left(\chi^{2}=6.44, p=0.092\right)$, patients with WHO PS 0 or $1+\left(\chi^{2}=2.80, p=0.423\right.$ and $\left.\chi^{2}=3.87, p=0.276\right)$, nor in early or metastatic disease patients $\left(\chi^{2}=1.50, p=0.682\right.$ and $\chi^{2}=3.32, p=0.345$, respectively). There was however some evidence to suggest a difference in least preferred questionnaire in patients with advanced local stage disease $\left(\chi^{2}=7.43, p=0.059\right)$, with $43 \%(16 / 37)$ of these patients choosing the EORTC QLQ-GI.NET21 questionnaire (Appendix: Table 3).

Principal investigators in each participating centre were contacted to ascertain their views on the use of QoL tools. None of the respondents used QoL tools in their routine practice but some had experience of their use in previous thyroid cancer trials. The majority of respondents felt that QoL tools could help patients explain and discuss their concerns, highlight issues that may other- 
wise be overlooked during a consultation, quantify QoL issues and demonstrate improving or declining trends in QoL. Investigators reported that information derived from QoL tools may be of most use to them when monitoring patients with advanced disease, who were likely to be symptomatic, and to monitor patients on systemic therapy. Finally, it was considered that use of such tools will aid both our understanding of the disease impact of MTC on patients and serve as a guide for future research.

\section{Discussion/Conclusion}

This is the first study to compare different QoL tools in patients with MTC. During the design stage of the study, there was the expectation that thyroid-specific questionnaires may be preferred overall and for those patients with locally advanced or metastatic disease, where there is a heavy disease burden and the likelihood that diarrhoea may be a troublesome symptom, that the EORTC NET questionnaire maybe of most value. The study findings, however, did not support this.

A significant number of participants responded that all questionnaires were useful, none were useful or left the preferences blank despite the protocol requiring each participant to provide their most and least preferred questionnaire. This may represent the patients' difficulties in deciding between the options with questions being preferred on different questionnaires.

There was evidence of a difference detected in the least preferred questionnaire with the EORTC NET questionnaire selected most often. This may reflect the patient population studied as the majority were of good performance status with early or locally advanced disease rather than metastatic disease. The questions for these subgroups, who were likely to be asymptomatic, may have proven to be least relevant for this time point in their cancer journey. A different finding may have occurred had the study included more symptomatic metastatic patients as this patient cohort may have been experiencing troublesome diarrhoea symptoms. Future studies should restrict the study population to those with progressive or metastatic disease, where QoL issues are more likely to be manifest and assessed.

If the QaLM study had shown a clear preference for any of the QoL tools, it was anticipated that this would then have been promoted in the thyroid oncology community as the preferred tool for use in routine practice, particularly for the cohort of patients on systemic ther- apy, and to monitor patients in the context of future interventional studies. One aspect this study has not fully addressed is patients' views on whether any of the QoL tools were fit for purpose and relevant to them in helping describe their QoL in relation to MTC-specific problems.

\section{Acknowledgements}

The authors would like to thank Claire Arthur, Kate Garcez, Nick Reed, Matt Beasley, Thomas Chambers, Vanessa Gill, Georgina Gerrard, James Good, Amy Roy and Mary Lei for their support in recruiting patients.

The authors would also like to thank Sam Morgan for his assistance in setting up the study sites and all the patients who took part.

\section{Statement of Ethics}

Subjects have given their written informed consent. The study protocol was approved by the Frenchay Research Ethics Committee and was listed on www.clinicaltrials.gov (NCT02465424).

\section{Disclosure Statement}

The authors have no conflicts of interest to declare.

\section{Funding Sources}

The study was supported by a research grant awarded by the Association for Multiple Endocrine Neoplasia Disorders (AMEND). The AMEND charity had no role in protocol development, data analysis or manuscript production.

\section{Author Contributions}

Laura Moss was the chief investigator and therefore involved with study design and development, patient recruitment, data collation, manuscript writing and review. The following authors were involved in study development, design, recruitment and manuscript review: Jonathan Wadsley, Kate Newbold and Mark Strachan. The following authors were involved in recruitment, study feedback and manuscript review: Maralyn Druce, Neil Tolley, Kathryn Graham, Sarah Jefferies, Lydia Fresco, Suganya Sivabalasingham and Alistair Balfour. Catrin Cox and Chris Hurt were involved in statistical design and analysis as well as manuscript review. 


\section{Appendix 1}

Characteristics of patients with and without most preferred questionnaire data

\begin{tabular}{|c|c|c|c|c|}
\hline \multirow[t]{2}{*}{ Characteristic } & \multicolumn{2}{|c|}{$\begin{array}{l}\text { Patients with data on their most } \\
\text { preferred questionnaire }(n=112)\end{array}$} & \multicolumn{2}{|c|}{$\begin{array}{l}\text { Patients without data on their most preferred } \\
\text { questionnaire }(n=92)\end{array}$} \\
\hline & $n$ & $\%$ & $n$ & $\%$ \\
\hline \multicolumn{5}{|l|}{ Gender } \\
\hline Male & 48 & 43 & 41 & 45 \\
\hline Female & 61 & 54 & 45 & 49 \\
\hline Missing & 3 & 3 & 6 & 7 \\
\hline \multicolumn{5}{|l|}{ Age } \\
\hline Median (IQR; range) & & $68 ; 22-82)$ & & $58(50-68 ; 24-80)$ \\
\hline Missing & 15 & 13 & 16 & 17 \\
\hline \multicolumn{5}{|l|}{ WHO PS } \\
\hline 0 & 68 & 61 & 64 & 70 \\
\hline 1 & 23 & 21 & 11 & 12 \\
\hline 2 & 4 & 4 & 3 & 3 \\
\hline 3 & 2 & 2 & 0 & 0 \\
\hline Missing & 15 & 13 & 14 & 15 \\
\hline \multicolumn{5}{|l|}{ Medullary thyroid cancer } \\
\hline Inherited MTC & 16 & 14 & 16 & 17 \\
\hline MEN type $2 A$ & 13 & 81 & 16 & 100 \\
\hline MEN type $2 B$ & 1 & 6 & 0 & 0 \\
\hline Familial MTC & 2 & 13 & 0 & 0 \\
\hline Sporadic & 77 & 69 & 60 & 65 \\
\hline Missing & 19 & 17 & 16 & 17 \\
\hline \multicolumn{5}{|l|}{ Tstage } \\
\hline 1 & 18 & 16 & 14 & 15 \\
\hline 2 & 28 & 25 & 19 & 21 \\
\hline 3 & 26 & 23 & 17 & 18 \\
\hline 4 & 9 & 8 & 2 & 2 \\
\hline $\mathrm{X}$ & 14 & 13 & 23 & 25 \\
\hline Missing & 17 & 15 & 17 & 18 \\
\hline \multicolumn{5}{|l|}{ N stage } \\
\hline 0 & 35 & 31 & 19 & 21 \\
\hline 1 & 1 & 1 & 2 & 2 \\
\hline $1 \mathrm{~A}$ & 10 & 9 & 9 & 10 \\
\hline 1B & 42 & 38 & 35 & 38 \\
\hline $\mathrm{X}$ & 7 & 6 & 10 & 11 \\
\hline Missing & 17 & 15 & 17 & 18 \\
\hline \multicolumn{5}{|l|}{ Disease stage } \\
\hline Early (T1-3, N0 or N1A) & 42 & 38 & 27 & 29 \\
\hline Advanced local (T4, any T N1b) & 28 & 25 & 30 & 33 \\
\hline Metastatic (any T, any N, M1) & 24 & 21 & 20 & 22 \\
\hline Missing & 18 & 16 & 15 & 16 \\
\hline
\end{tabular}




\section{Appendix 2}

Patients' most and least preferred QoL tools

\begin{tabular}{|c|c|c|c|c|}
\hline \multirow{3}{*}{ Quality of Life Questionnaires } & \multirow{2}{*}{\multicolumn{2}{|c|}{$\begin{array}{l}\text { Most preferred data cohort }(n=112) \\
\text { most preferred }\end{array}$}} & \multirow{2}{*}{\multicolumn{2}{|c|}{$\begin{array}{l}\text { Least preferred data cohort }(n=128) \\
\text { least preferred }\end{array}$}} \\
\hline & & & & \\
\hline & $n$ & $\%$ & $n$ & $\%$ \\
\hline EORTC QLQ-C30 & 30 & $31 \%$ & 22 & $23 \%$ \\
\hline MDASI-Thy & 21 & $22 \%$ & 21 & $22 \%$ \\
\hline City of Hope: QoL-Thy & 23 & $24 \%$ & 23 & $24 \%$ \\
\hline EORTC QLQ-GI.NET21 & 22 & $23 \%$ & 30 & $31 \%$ \\
\hline$p$ value & & 0.650 & & 0.042 \\
\hline
\end{tabular}

\section{Appendix 3}

Patients' most preferred and least preferred QoL tools by subgroup

\begin{tabular}{|c|c|c|c|c|c|c|c|c|c|}
\hline & \multicolumn{2}{|c|}{$\begin{array}{l}\text { EORTC } \\
\text { QLQ-C30 }\end{array}$} & \multicolumn{2}{|c|}{ MDASI-Thy } & \multicolumn{2}{|c|}{$\begin{array}{l}\text { City of Hope: } \\
\text { QoL-Thy }\end{array}$} & \multicolumn{2}{|c|}{$\begin{array}{l}\text { EORTC } \\
\text { QLQ-GI.NET21 }\end{array}$} & \multirow[t]{2}{*}{$p$ value } \\
\hline & $n$ & $\%$ & $n$ & $\%$ & $n$ & $\%$ & $n$ & $\%$ & \\
\hline \multicolumn{10}{|l|}{ Type of medullary thyroid cancer } \\
\hline \multicolumn{10}{|l|}{ Most preferred } \\
\hline Inherited MTC $(n=16)$ & 9 & 56 & 2 & 13 & 1 & 6 & 4 & 25 & _* \\
\hline Sporadic MTC $(n=77)$ & 17 & 22 & 16 & 21 & 21 & 27 & 23 & 30 & 0.637 \\
\hline \multicolumn{10}{|l|}{ Least preferred } \\
\hline Inherited MTC $(n=18)$ & 3 & 17 & 2 & 11 & 6 & 33 & 7 & 39 & _* \\
\hline Sporadic MTC $(n=90)$ & 19 & 21 & 23 & 26 & 16 & 18 & 32 & 36 & 0.092 \\
\hline \multicolumn{10}{|l|}{ WHO performance status } \\
\hline \multicolumn{10}{|l|}{ Most preferred } \\
\hline $0(n=68)$ & 20 & 29 & 15 & 22 & 17 & 25 & 16 & 24 & 0.844 \\
\hline $1+(n=29)$ & 6 & 21 & 6 & 21 & 8 & 28 & 9 & 31 & 0.818 \\
\hline \multicolumn{10}{|l|}{ Least preferred } \\
\hline $0(n=81)$ & 18 & 22 & 21 & 26 & 16 & 20 & 26 & 32 & 0.423 \\
\hline $1+(n=30)$ & 7 & 23 & 5 & 17 & 6 & 20 & 12 & 40 & 0.276 \\
\hline \multicolumn{10}{|l|}{ Disease stage } \\
\hline \multicolumn{10}{|l|}{ Most preferred } \\
\hline Early stage disease $(n=42)$ & 12 & 29 & 4 & 10 & 12 & 29 & 14 & 33 & 0.132 \\
\hline Advanced local stage disease $(n=28)$ & 4 & 14 & 9 & 32 & 9 & 32 & 6 & 21 & 0.463 \\
\hline Metastatic disease $(n=24)$ & 7 & 29 & 8 & 33 & 3 & 13 & 6 & 25 & 0.506 \\
\hline \multicolumn{10}{|l|}{ Least preferred } \\
\hline Early stage disease $(n=48)$ & 9 & 19 & 14 & 29 & 11 & 23 & 14 & 29 & 0.682 \\
\hline Advanced local stage disease $(n=37)$ & 9 & 24 & 7 & 19 & 5 & 14 & 16 & 43 & 0.059 \\
\hline Metastatic disease $(n=25)$ & 5 & 20 & 4 & 16 & 6 & 24 & 10 & 40 & 0.345 \\
\hline
\end{tabular}

* Test assumptions not met. 


\section{References}

1 Cancer Research UK. https://www.cancerresearchuk.org/health-professional/cancerstatistics/statistics-by-cancer-type/thyroidcancer.

2 Hundahl SA, Fleming ID, Fremgen AM, Menck HR. A National Cancer Data Base report on 53,856 cases of thyroid carcinoma treated in the U.S., 1985-1995. Cancer. 1998 Dec;83(12):2638-48.

3 Pelizzo MR, Boschin IM, Bernante P, Toniato A, Piotto A, Pagetta C, et al. Natural history, diagnosis, treatment and outcome of medullary thyroid cancer: 37 years experience on 157 patients. Eur J Surg Oncol. 2007 May;33(4):493-7.
4 Aaronson NK, Ahmedzai S, Bergman B, Bullinger M, Cull A, Duez NJ, et al. The European Organization for Research and Treatment of Cancer QLQ-C30: a quality-of-life instrument for use in international clinical trials in oncology. J Natl Cancer Inst. 1993 Mar;85(5):365-76.

5 Gning I, Trask PC, Mendoza TR, Harle MT, Gutierrez KA, Kitaka SA, et al. Development and initial validation of the thyroid cancer module of the M. D. Anderson Symptom Inventory. Oncology. 2009;76(1):59-68.
6 Ferrell BR, Dow KH, Grant M; City of Hope. Measurement of the quality of life in cancer survivors. Qual Life Res. 1995 Dec;4(6):52331.

7 Yadegarfar G, Friend L, Jones L, Plum LM, Ardill J, Taal B, et al; EORTC GINET21. Validation of the EORTC QLQ-GINET21 questionnaire for assessing quality of life of patients with gastrointestinal neuroendocrine tumours. Br J Cancer. 2013 Feb;108(2): 301-10. 